\title{
Study of $\mathrm{Si}(100)-\mathrm{SiO}_{2}$ Interface Trap Time Constant Distribution in Large Area Conventional MOSFETs - Comparison with Submicron Devices
}

\author{
D. Bauza ${ }^{a}$ and N. Guenifi ${ }^{a, b}$ \\ ${ }^{a}$ IMEP-LaHC, Minatec, Grenoble-INP, 3 Parvis Louis Néel, CS 50257, 38016, \\ Grenoble Cedex 1 France. \\ ${ }^{\mathrm{b}}$ LEA Electronic Department, University of Mostefa Benboulaid, Batna, Algeria.
}

A charge pumping (CP) technique has been proposed more than two decades ago to extract the $\mathrm{Si}(100)-\mathrm{SiO}_{2}$ interface trap time constant distribution (ITTCD) that exists at this interface. To that aim, several Elliot curves (1) needed to be recorded at a frequency, $\mathrm{f}_{0}$, of the order of $\mathrm{f}_{0}=10^{4} \mathrm{~Hz}$ and for a set of selected gate voltage swing, Vsw, values. The charge recombining during one period of the gate signal, $\mathrm{Qcp}_{0}=\mathrm{Icp}_{0} / \mathrm{f}_{0}$ was extracted from the maximum CP current measured, $\mathrm{Icp}_{0}$, at $\mathrm{f}_{0}$, supposed to result from recombination due to identical electron and hole free carrier concentrations at the interface. From $\mathrm{Qcp}_{0}$, the recombined charge was finally measured as function of frequency, $f$, yielding a set of $\mathrm{Qcp}_{0}(\mathrm{f})$ curves, one for each Vsw value. Assuming, as done primarily, that carrier capture resulted from tunneling from the interface to the traps in the near oxide, the set of $\mathrm{Qcp}_{0}(\mathrm{f})$ curves provided a single trap "depth" concentration profile. The same kind of profile was recorded from n- and p-channel MOSFETs of different technologies (2, 3). As $\mathrm{Pb} 0$ centers are known to be the amphoteric defects that characterize the $\mathrm{Si}(100)-\mathrm{SiO}_{2}$ interface, even after forming annealing in conventional MOSFETs with thermally grown oxides, and as these centers are located at the interface, the trap time constant distribution was re-interpreted. Then, in order to evidence the way the $\mathrm{Pb} 0$ centers occupy the interface, the experimental conditions primarily used to extract the profiles are modified. These results are discussed with regard to previous work in that field $(\mathbf{4}, \mathbf{5})$ and to recent results obtained by $\mathrm{T}$. Tsuchiya and co-workers on submicron devices $(6,7)$.

\section{Introduction}

The $\mathrm{Si}(100)-\mathrm{SiO}_{2}$ system strongly contributed to the success of MOSFET's technology and the $\mathrm{Si}(100)-\mathrm{SiO}_{2}$ interface that possesses better interface properties than those obtained using other silicon orientations attracted much attention during the past decades. Nevertheless, in spite of years of fruitful research, basic points remain unclear or require deeper investigations. Among them detailed properties of the $\mathrm{Pb} 0$ centers, those traps that characterize this interface even after the "forming gas annealing" traditionally carried out at the end of the technological process on MOSFETs with thermally grown oxides. In this paper, the charge pumping (CP) technique initially proposed to extract the $\mathrm{Si}(100)-\mathrm{SiO}_{2}$ Interface Trap Time Constant Distribution (ITTCD) assuming tunneling for carrier capture 
is discussed. Specific experimental conditions are used to point out the trap properties underlying the obtained trap profiles. Results in that field are reported and discussed with regard to those obtained previously. The measurements do not show evidence of the other amphoteric defects, the $\mathrm{Pb} 1$ centers which may share this interface with the $\mathrm{Pb} 0$ ones, the presence and electrical activity of which have been questioned $(\mathbf{8})$.

\section{Initial extraction of the Interface Trap Time Constant Distribution (ITTCD)}

Its principle has been briefly depicted in the abstract. When this approach was proposed (9), one was far from the knowledge acquired until then on $\mathrm{Si}_{-} \mathrm{SiO}_{2}$ interface properties. Besides, with the shrinking during the 90 's of the $\mathrm{SiO}_{2}$ thickness, the existence of slow traps and their impact on devices low frequency noise (10) as well as the increase in gate leakage current when the $\mathrm{SiO}_{2}$ layers became thinner than $3 \mathrm{~nm}$ became topical (11). The primary extraction of the $\mathrm{Si}(100)-\mathrm{SiO}_{2}$ Interface Trap Time Constant Distribution (ITTCD) was carried out by recording first, and at a given frequency, typically $10^{4} \mathrm{~Hz}$, Elliot curves using gate voltage swing, Vsw, values typically equal to $0.6 \mathrm{~V}, 0.8 \mathrm{~V}, 1 \mathrm{~V}, 1.5 \mathrm{~V}, 2 \mathrm{~V}$ and when necessary, $2.5 \mathrm{~V}$ and $3 \mathrm{~V}$. Here, $\mathrm{Vsw}=(\mathrm{Vh}-\mathrm{Vl})$, where $\mathrm{Vh}$ is the high value of the gate signal, $\mathrm{Vg}$, which biases the device toward inversion (n-channel MOSFET case), Vl being the low Vg value that biases it toward accumulation. These CP curves were measured starting from Vl in accumulation in a region where the CP current, Icp, is small, up to a region where $\mathrm{Vh}$ is in strong inversion and where Icp approaches again zero. Such bell-shaped curves, also called Elliot curves (1) scan the silicon bandgap at a given frequency and for a given Vsw value so that all the regions in which Icp can be recorded, i.e. in which recombination is significant are accounted for. Then, and as far as the channel of the MOSFET is uniform (12), Icp should bring information on the traps underneath. Repeated using the successive Vsw values noted above, following the steps mentioned in the abstract and provided that the $\mathrm{SiO}_{2}$ layer was thicker than a few nanometers yielded a set of $\mathrm{Qcp}_{0}(\mathrm{f})$ curves as function of frequency, corresponding to as much as $\mathrm{Qcp}_{0}(\mathrm{f})$ curves as there was Vsw ones $(\mathbf{1 1}, \mathbf{1 2})$.

Then, the so-obtained $\mathrm{Qcp}_{0}(\mathrm{f})$ curves were plotted assuming that electron and hole capture cross sections decrease exponentially from the interface toward oxide depth according to the simple tunneling model:

$$
\mathrm{xe}=\lambda \mathrm{e} \ln [\mathrm{ns} \sigma \mathrm{e}(0) \mathrm{vth} \text { th }] \quad \text { and } \quad \mathrm{xh}=\lambda \mathrm{h} \ln [\mathrm{ps} \sigma \mathrm{h}(0) \mathrm{vth} \mathrm{tl}]
$$

In Eq. 1, xe and xh are the oxide depth reached by electrons during the time th in inversion and by holes during that, $\mathrm{tl}$, in accumulation. $\lambda \mathrm{e}$ and $\lambda \mathrm{h}$ are the tunnel attenuation length for electrons and holes, respectively, taken equal, i.e $\lambda \mathrm{e}=\lambda \mathrm{h}=$ $\lambda$ eh. ns and ps are the electron and hole concentration at the interface during th and tl, respectively, $\sigma \mathrm{e}(0)$ and $\sigma \mathrm{h}(0)$ being the trap capture cross section for electrons and holes at the interface. vth is the carriers thermal velocity. For all Vsw values, Icpmax was supposed to result from recombination up to $\mathrm{xr}$ such that $\mathrm{xe}=\mathrm{xh}=\mathrm{xr}$.

By doing so, the $\mathrm{Qcp}_{0}(\mathrm{f})$ curves obtained superimpose remarkably well in the regions where they overlap, the same "xr" through different Vsw and f values yielding an overall profile accounting for the $\mathrm{Si}(100)-\mathrm{SiO}_{2}$ Interface Trap Time Constant Distribution (ITTCD) extracted in such conditions $(\mathbf{1 1}, \mathbf{1 2})$. 
After correcting the profiles close to the interface, where the filling function variation, $\Delta \mathrm{F}(\mathrm{x})$, becomes smaller than unity $(\mathbf{9}, \mathbf{1 2})$, an exponential decrease in the trap concentration from the interface toward oxide depth was obtained followed by a plateau attributed to the traps in the near oxide, as verified by noise spectroscopy on the devices studied (12), and as also measured using other approaches such as the conductance technique extended toward low frequencies (13). For fresh devices the trap density at the interface is in the order of 100 to 200 times that in the slow traps plateau region.

Further at negative " $x$ " values (i.e. toward silicon), a plateau was also expected accounting for the high frequency limit of the $\Delta \mathrm{F}(\mathrm{x})$ function given by:

$$
(\text { cn.cp }) /(\mathrm{cn}+\mathrm{cp})
$$

In Eq. 2, cn and cp are the capture rate for electrons and holes, respectively, in such small Vsw conditions. They are given by $\mathrm{cn}=\mathrm{ns} . \sigma \mathrm{e}(0)$.vth and $\mathrm{cp}=\mathrm{ps} \sigma \mathrm{h}(0)$ vth $(\mathbf{5 , 1 0 , 1 2})$. This plateau was also observed when the interface correction was applied (12).

Similar profiles have been extracted from $\mathrm{n}$ - and $\mathrm{p}$-channel MOSFETs of different technologies $(\mathbf{2}, \mathbf{1 2})$, and from $\mathrm{n}$ - and p-channel MOSFETs having thin RTCVD oxinitride films as gate dielectric (3). When these "profiles" were compared to those recorded from Current Transient (CT) measurements a qualitative agreement was noted (3). Generally, the CT profiles having a smaller slope than the exponential part of the CP ones, they were crossing them (17).

As discussed above and in addition to the remarkable extraction of a single trap profile from the set of Qcp 0 (f) curves obtained using different Vsw values, the profiles extracted provided reliable oxide trap concentrations in the plateau region accounting for near oxide traps. The expected behavior at the interface with the partial filling of the traps through the $\Delta \mathrm{F}(0)$ correction along with the plateau due to the high frequency limit of the same $\Delta \mathrm{F}$ function were also verified. These profiles have been shown to be independent of the gate signal transition times, $\operatorname{tr}$ and $\mathrm{tf}$, in a range of tr and tf values from $50 \mathrm{~ns}$ to $300 \mathrm{~ns}$. Integration over depth of the trap concentration gave interface trap densities, Dit, values close to those measured using conventional Dit extraction CP method (14).

However, once these properties established, the mechanism for carrier capture was questioned (12) and besides the $\mathrm{Pb} 0$ centers, the amphoteric defects that exist at this interface, even after forming gas annealing in case of conventional MOSFETs with thermally grown oxides, several points supported that the properties leading to these profiles were not really understood yet.

The main one is the systematic but fruitful use of tunnel attenuation lengths equal for electrons and holes, $\lambda \mathrm{e}=\lambda \mathrm{h}=\lambda \mathrm{eh}$, as already noted. One may expect these values to differ in the case of slow traps toward oxide depth but not for the whole profiles. This point was further evidenced when simulating for the first time and very accurately the three basic CP curve types gathered by Groeseneken et al. in (14) in their well-known paper $(\mathbf{1 5}, \mathbf{1 6})$. Furthermore, CP curves simulations were not able to account for the actual CP Elliot curves recorded using Vsw values smaller than $\mathrm{Vsw}=0.6 \mathrm{~V}$, for instance at $\mathrm{Vsw}=0.4 \mathrm{~V}$, in which two components entering the CP signal were always observed, as can be seen in Fig. 25 (a) in (18), one on the left hand side of the CP signal, the other on its right hand side. Among 
the devices studied in that field none of these components was systematically greater than the other one in such conditions. Increasing Vsw beyond $0.4 \mathrm{~V}$ lead these components to merge yielding a $\mathrm{CP}$ signal more or less symmetrical and roughly simulated $(\mathbf{1 5}, \mathbf{1 6})$.

\section{Wachnik and Lowney's approach}

Such signals were primarily pointed out a long time ago by R. A. Wachnik and J. R. Lowney $(4,5)$ who applied small pulse heights on the $\mathrm{Si}(100)-\mathrm{SiO}_{2}$ interface of their devices using the Small Rectangular Pulse (SRP) method they proposed $(\mathbf{4}, \mathbf{5})$. In this technique, the gate signal transition times are short enough to prevent carrier emission to occur during them. They therefore proposed a filling function variation, $\Delta \mathrm{F}$, assuming traps located at the interface. Using pulse heights well below those used in the large gate pulse mode (14-16) called by the authors the "transition time technique", they considered that in such conditions, the traps observed, assumed to have two charge states, charged and uncharged, only exchange carried by capture (5).

Then, small gate pulses of slightly increasing Vsw values (but always smaller than $0.4 \mathrm{~V}$ ) were applied to their devices such that the average voltage called the offset voltage situates the Fermi level close to midgap (5). The basic idea was to study the traps located there.

In a second time, starting from this point, Icp was recorded as a function of frequency using the same set of pulse amplitudes. In both cases, the measurements were compared with what was expected from the model derived to that aim, accounting for surface potential fluctuations and carrier capture through the filling function variation, $\Delta \mathrm{F}$.

Because only capture was supposed to occur during the measurements, the authors treat the traps observed as specific components of the overall interface trap density measured using the large gate pulse method (14).

In the case of dry oxidation and after forming gas annealing, there was no evidence that two trap types entered the curve measured but the fitting of the curves was improved by using the author's two traps model (4). When oxidation was carried out in pyrogenic steam, the CP signal clearly showed two components, before as well as after irradiation by gamma rays $(\mathbf{5})$.

As $\mathrm{Pb} 0$ centers characterize the $\mathrm{Si}(100)-\mathrm{SiO}_{2}$ interface, it is likely that the traps the authors observed in the central region of the silicon bandgap were the same as those we observe using Vsw values smaller than $0.6 \mathrm{~V}$, i.e. the acceptorand donor-like components of these amphoteric centers. The possible presence of such traps in the measurements was envisaged in (5). The author noted that a specific statistics should be required in such a case.

\section{Interface Trap Time Constant Distribution (ITTCD): traps located at the interface}

The final form of the trap profiles when assuming tunneling for carrier capture has been given in the above paragraph, i.e. an exponential decrease in trap concentration from the interface toward oxide depth followed by a plateau due slow or near oxide traps, in agreement with noise spectroscopy when this 
comparison was carried out (12). In this approach, the largest part of the interface traps was located within the first atomic layers from the interface.

With regard to the ITTCD, a first attempt to dissociate possible contributions to the overall CP signal was carried out a few years ago (19). This was done by verifying the reliability of the initial model in which recombination occurring up to $\mathrm{xr}$ resulted from electron and hole capture up to identical depths such that $\mathrm{xe}=$ $\mathrm{xh}=\mathrm{xr}$ (Eq. 1). As soon as this condition was not satisfied anymore, $\mathrm{xr}$ should obey $\mathrm{xr}=\min (\mathrm{xe}, \mathrm{xh})$. This was carried out using asymmetrical capture conditions.

Then, using Vsw $=0.8 \mathrm{~V}$, a value such that the two components visible at $\mathrm{Vsw}=0.4 \mathrm{~V}$ already merged, and starting from a gate signal at $\mathrm{f}=10^{6} \mathrm{~Hz}$ in which $\mathrm{th}=\mathrm{tl}$, th was increased with regard to tl on the one hand, or tl was increased with regard to th on the other hand. In other words starting from a small but identical filling of the traps by the two carrier types, the filling by one carrier type was increased with regard to the other and vice versa. According to the model, in both cases, the shorter filling time should control and fully limit recombination.

Similarly, starting now from a gate signal of a lower frequency, say $10^{3} \mathrm{~Hz}$, with th $=$ tl, i.e. from a significant filling of the traps by the two carrier types with the corresponding recombination, tl was decreased with regard to th or th was decreased with regard to tl. Then, and according again to the model, reducing one time with regard to the other, should allow this time to control recombination and the same trap profile should be obtained either through th or tl reduction.

In all cases and contrary to what was expected a $\mathrm{CP}$ current was measured so that a simple model accounting for now the presence of two trap types, acceptorlike traps the filling of which was controlled by th and donor-like ones filled through $\mathrm{tl}$, was proposed. Then, starting from a gate signal at $10^{6} \mathrm{~Hz}$ with $\mathrm{tl}=\mathrm{tl}$ and increasing th with regard to tl should fill the acceptor-like traps, while keeping the filling of the donor- like ones constant. Similarly, increasing tl with regard to th would fill the donor-like traps, while keeping the filling of the acceptor-like ones constant.

Starting now from $\mathrm{f}=10^{3} \mathrm{~Hz}$ with th $=\mathrm{tl}$ and reducing th with regard to tl should limit the acceptor-like traps filling, providing the profile of these trap type while reducing now tl with regard to th should reduce the donor-like traps filling and provide this trap type profile.

Applied to the results this model was very well verified but provided additional and unexpected results. In short and in all cases, the relative variations measured when reducing one time with regard to the other and vice versa provided the $\mathrm{CP}$ variations obtained by varying the gate signal frequency, supporting that the overall trap profiles extracted primarily were the sum of the two contributions.

Increasing or decreasing tl with regard to th yielded the same profile attributed to the donor-like component of the measured defects with a nearly perfect superimposition of the profiles in the regions where they overlap. This contribution was crossing the interface as defined by the initial frequency profile before falling off, supporting the donor-like contribution to be main one at Vsw = $0.4 \mathrm{~V}$. More surprisingly and concerning the acceptor-like component, starting from $\mathrm{f}=10^{3} \mathrm{~Hz}$, the trap concentration profile measured when reducing th with 
regard to tl provided twice the concentrations obtained when increasing it when starting from $10^{6} \mathrm{~Hz}$.

Indeed, at $\mathrm{f}=10^{6} \mathrm{~Hz}$, donor-like traps are only lightly filled so that increasing the filling of the acceptor-like traps through th only provides the acceptor-like profile. On the contrary, when starting from $\mathrm{f}=10^{3} \mathrm{~Hz}$, both trap types are significantly filled. In such conditions the acceptor-like trap concentrations were multiplied by two when recorded in the presence of filled donor-like traps, suggesting that $\mathrm{Pb} 0$ centers with their two components were probed in such small Vsw conditions.

The device studied in (19) underwent a Fowler-Nordheim injection from the gate of $10^{17} \mathrm{q} \cdot \mathrm{cm}^{-2}$. This result may be understood in the light of the Spin Dependent Recombination (SDR) measurements carried out in (20) which showed that after irradiation, $\mathrm{Pb} 0$ centers are present down to the center of the silicon bandgap.

Finally, and as noted above, when the trap profile of the donor-like component recorded in such asymmetrical conditions was measured beyond the decline in trap density recorded at the interface when varying the gate signal frequency, the decrease in trap density of the acceptor-like traps profile was visible at small positive $\mathrm{x}$ value, confirming the shift in trap density and probably in capture cross section between the donor-like and the acceptor-like components, this later one being very probably the smaller one in the signal observed on this device at Vsw $=0.4 \mathrm{~V}(\mathbf{1 9})$.

The properties pointed put in the preceding paragraphs when measured using $\mathrm{Vsw}=0.8 \mathrm{~V}$ were also observed at $\mathrm{Vsw}=1 \mathrm{~V}$.

The recent work by T. Tsuchiya end co-workers on submicron devices

Recently, CP signals Icp = 2.q.f in height corresponding to a charge recombining Qcp $=\mathrm{Icp} / \mathrm{f}=2$.q in a single step were measured on devices of length 100 to $130 \mathrm{~nm}$ and width 110 to $200 \mathrm{~nm}$ having $4 \mathrm{~nm}$ thick silicon oxides grown by Rapid Thermal Oxidation (RTO). Such CP signals were extracted from a large number of signals, ranging from Icp $<<$ f.q to Icp $>>$ f.q but resulting from several more or less simultaneous ( f.q) events having various heights. The interface trap density, Dit, of the devices was in the $5.10^{10} \mathrm{eV}^{-1} \cdot \mathrm{cm}^{-2}-10^{11} \mathrm{eV}^{-1} \cdot \mathrm{cm}^{-2}$ range $(6,7)$.

Varying the parameters of the gate signal applied to their devices, the authors classify the contributions they observed into different positions in the Si bandgap of the acceptor- and donor-like components of the $\mathrm{Pb} 0$ amphoteric centers which are typically supposed to be located at $\mathrm{Ei} \pm 0.3 \mathrm{eV}$. As this energy width, $\Delta \mathrm{E} \approx 0.6 \mathrm{eV}$, is the energy $\mathrm{CP}$ can probe at room temperature at the $\mathrm{Si}_{-} \mathrm{SiO}_{2}$ interface, the authors note that detecting these centers in a single $\mathrm{CP}$ signal was not easy as pointed out below.

Nevertheless, in view of clean (2.f.q) CP signals, the authors unambiguously recognized the signatures of single $\mathrm{Pb} 0$ centers, observed here for the first time. Indeed, the first single transition events (i.e. f.q. signals) recorded using CP from submicron MOSTETs of a $0.5 \mu \mathrm{m}$ CMOS technology dated from 1986 (21). 
To explain the wide range of $\mathrm{CP}$ signals measured with regard to the few $\mathrm{Pb} 0$ centers fully detected, the authors classified the acceptor-like and donor-like components of the centers according to their energy position in the Si bandgap with regard to the energy swept there by $\mathrm{CP}$. The energy position of the components may be determined by varying the transition times of the gate signal. One of the components may have a capture time constant longer than the time th or tl available for capture. Coulomb interactions may also impact trap detection (7).

\section{Trap profiles and interface trap time constant distribution (ITTCD)}

From the studies carried out in (19), pointing out the presence at the interface of the two components of the $\mathrm{Pb} 0$ centers, it was clear that the tunneling approach of the ITTCD was not justified. Instead, the expression of the ITTCD in terms of simple trap time constant distribution at the interface is right. Then increasing Vsw by steps allows more interface traps to enter recombination regardless of the peak contributions of the acceptor- and donor-like components of the $\mathrm{Pb} 0$ defects, leading to an increase in the $\mathrm{CP}$ recombination current. The maximum $\mathrm{CP}$ current recorded at a given Vsw results from to identical contributions of acceptor- and donor like components of those centers through the comparison at a given Vsw between the capture rates and the time for capture, that is (cn.th) and (cp.tl) accounted for in the $\Delta \mathrm{F}$ function.

In this presentation, the various contributions depicted in the preceding and that permitted to improve our understanding of the $\mathrm{Si}(100)-\mathrm{SiO}_{2}$ interface and the charge pumping curves that can be extracted from it will be recalled. The trap profiles accounting for the presence there of $\mathrm{Pb} 0$ centers will be depicted. The impact of asymmetrical capture conditions, as primarily reported in (19) in order to point out the $\mathrm{Pb} 0$ components in the $\mathrm{CP}$ signal will be presented and discussed.

Device studied. The device studied in this work is an n-channel MOSFET fabricated at IBM Microelectronics (Corbeil-Essonnes, France) using a $0.5 \mu \mathrm{m}$ CMOS technology. The source and the drain of the device form a single combshaped junction with the substrate that interpenetrates the polysilicon gate comb. The large area of the device, $20.000 \mu \mathrm{m}^{2}$ gives short effective channel length of $0.33 \mu \mathrm{m}$. The oxide thickness is $13 \mathrm{~nm}$, the doping concentration is $1.210^{17} \mathrm{~cm}^{-3}$. These devices exhibit low interface trap densities, in the low $10^{9} \mathrm{eV}^{-1} \cdot \mathrm{cm}^{-3}$ range.

\section{References}

1. A.B.M. Elliot, Solid-State Electron. 19, 241, (1979).

2. D. Bauza and Y. Manéglia, IEEE Trans. Electron Devices ED-44, 2262 (1997).

3. Y. Maneglia, D. Bauza, Y. Morfouli, and G. Ghibaudo, Microelectronic Engineering, 36, 153 (1997).

4. R.A. Wachnik and J.R. Lowney, Solid-St. Electron. 29, 447 (1986).

5. R.A. Wachnik, IEEE Trans. Electron Devices ED-33, 1054 (1986).

6. T. Tsuchiya and Y. Ono, Jap. J. Appl. Phys., 54, 04 DC01 (2015).

7. T. Tsuchiya and P.M. Lenahan, Jap. J. Appl. Phys., 56, 031301 (2017).

8. A. Stesmans and V.V. Afanas'ev, Physical Review B 57, 10030 ((1998).

9. D. Bauza and G. Ghibaudo, Solid-St. Electron., 39, 563 (1996).

10. D. Bauza and G. Ghibaudo, Microelectronic Engineering, 28, 325 (1995). 
11. D. Bauza, Solid-St. Electronics 47, 1677 (2003).

12. Y. Manéglia, F. Rahmoune, and D. Bauza, J. Appl. Phys., 97, 014502 (2004).

13. M.J. Uren, S. Collins, and M.J. Kirton, Appl. Phys. Lett,. 54, 1448 (1989).

14. G. Groeseneken, H. E. Maes, N. Beltran, and R. F. De Keersmaeker, IEEE Trans. Electron Devices, ED-31, 42 (1984).

15. D. Bauza, IEEE Trans. Electron Devices, ED-56, 70 and 78 (2009).

16. D. Bauza, IRPS 2013, Paper G.D. 2.1.

17. D. Bauza, J. Appl. Phys., 84, 6178 (1998).

18. D. Bauza, O. Ghobar, N. Guenifi, and S. Bayon, in "Silicon Nitride, Silicon Dioxide and Emerging Dielectrics," ECS Transactions Vol. 19 N 2 (2009).

19. Y. Manéglia and D. Bauza, in "Silicon Nitride, Silicon Dioxide thin Insulating Films", ECS Proceedings Volume PV 2005-1, pp. 33-44.

20. M.A. Jupina and P. M. Lenahan, IEEE Trans. Nuclear Science, 37, 1650 (1990).

21. G. Groeseneken I. De Wolf, R. Bellens, and H.E. Maes, IEEE Trans. Electron Devices ED-43, 940 (1986). 